pylori representing the majority of cases. In those with no apparent cause, there may be an association with IBS and the IEL count becomes normal on repeat biopsy in $71 \%$.

Competing interests None declared.

\section{OC-103 TUMOUR REGRESSION GRADING CORRELATES TO SURVIVAL IN OESOPHAGO-GASTRIC CANCER. A REPORT OF CURRENT SURVIVAL OUTCOMES FOLLOWING NEO-ADJUVANT CHEMOTHERAPY}

doi:10.1136/gutjnl-2012-302514a.103

${ }^{1}$ A M Reece-Smith,* ${ }^{2}$ S Soomro, ${ }^{3} S$ madhusudan, ${ }^{1} S$ L Parsons. ${ }^{1}$ Department of Oesophago-gastric surgery, Nottingham University NHS trust, Nottingham, UK; ${ }^{2}$ Department of Histopathology, Nottingham University NHS trust, Nottingham, UK; ${ }^{3}$ Department of Medical Oncology, Nottingham University NHS trust, Nottingham, UK

Introduction Patients who demonstrate a histological response to chemotherapy as determined by tumour regression grading (TRG) are known to have improved survival. ${ }^{1}{ }^{2}$ We report current survival expectations for patients following neo-adjuvant chemotherapy and resection of gastro-oesophageal cancer.

Methods TRG was determined by a specialist pathologist in 250 patients after chemotherapy, and survival outcomes were assessed by Kaplan-Meier analysis of patients divided into responder (Mandard TRG 1-3) and non-responder (TRG 4\&5) groups, with log-rank comparisons.

Results After a median follow-up of 54.4 months survival was found to be improved in those with histological response to chemotherapy $(p<0.001)$. Median survival was estimated to be 34.0 months and 21.2 months in oesophageal adenocarcinoma responders and non-responders and this was statistically significant $(p=0.050)$. Junctional cancers demonstrated the largest survival difference at 51.1 months compared to 24.6 months $(p=0.003)$. In gastric cancers the median survival was yet to be reached for responders and was 35.2 months for non-responders. However, smaller numbers in this group led to loss of statistical power $(p=0.166)$. A higher proportion of patients with good response to chemotherapy was not seen using MAGIC regimen (Pearson $\chi^{2}$ $p=0.616)$. Good response to chemotherapy (TRG1-3) was associated with lower incidence of positive CRM ( $p=0.021)$

Conclusion Tumour regression grading is a useful predictor of overall survival allowing clinicians greater ability to counsel their patients. However, MAGIC therapy appears to improve survival possibly through mechanisms other than simply increasing the proportion of patients that have histological response.

Abstract 0C-103 Table 1 Proportion of patients achieving which tumour regression grades following either MAGIC regimen chemotherapy (three cycles of neo-adjuvant $E C F / X$ ) or other regimens (non-MAGIC)

\begin{tabular}{|c|c|c|c|c|c|c|c|}
\hline TRG & & 1 & 2 & 3 & 4 & 5 & Total \\
\hline \multirow[t]{2}{*}{ Non-magic } & $\mathrm{n}=$ & 3 & 5 & 24 & 33 & 35 & 100 \\
\hline & $\%$ & $3.0 \%$ & $5.0 \%$ & $24.0 \%$ & $33.0 \%$ & $35.0 \%$ & $100.0 \%$ \\
\hline \multirow[t]{2}{*}{ Magic } & $\mathrm{n}=$ & 8 & 12 & 31 & 46 & 41 & 138 \\
\hline & $\%$ & $5.8 \%$ & $8.7 \%$ & $22.5 \%$ & $33.3 \%$ & $29.7 \%$ & $100.0 \%$ \\
\hline Total & & 11 & 17 & 55 & 79 & 76 & 238 \\
\hline
\end{tabular}

Competing interests None declared.

\section{REFERENCES}

1. Becker, et al. Ann Surg 2011;253:934-9

2. Fareed, et al. Histopathology 2009;55:399-406.
OC-104 THE VALIDITY OF THE ROYAL COLLEGE OF PATHOLOGISTS' STOMACH CANCER MINIMUM DATASET IN A POPULATION USING THE NORTHERN \& YORKSHIRE CANCER REGISTRY DATA

doi:10.1136/gutjnl-2012-302514a.104

${ }^{1} \mathrm{O}$ Rotimi, ${ }^{*}{ }^{1} \mathrm{H}$ Grabsch, ${ }^{2} \mathrm{E}$ Morris. ${ }^{1}$ Department of Histopathology, Leeds Teaching Hospitals Trust, Leeds, UK; ${ }^{2}$ NYCRIS, Leeds Teaching Hospitals Trust, Leeds, UK

Introduction Quality histopathological reporting of resceted stomach cancer provides important information for treatment of patients. Sub-standard reporting leads to incorrect staging which impacts on treatment and ultimately survival. Proforma reporting has been introduced as a means of standardisation. This study sought to validate the prognostic significance of the Royal College of Pathologists (RCPath) stomach cancer dataset ${ }^{1}$ in a population.

Methods A retrospective analysis of pathology forms from 1065 resected stomach cancer from 1995 to 2006 was carried out. The variables reported were related to NYCRIS registry survival data using univariate Kaplan-Meier method and Cox proportional regression modelling.

Results The study population was representative of the Yorkshire stomach cancer population. Survival was poorer with increasing age but it improved significantly from $29.0 \%$ (95\% CI $23.1 \%$ to $35.1 \%$ ) in $1995-1997$ period to $50.5 \%$ (95\% CI $40.1 \%$ to $60.1 \%)$ in the 2004-2006 period. Depth of local invasion, nodal stage and completeness of excision are validated as independent prognostic factors in a population setting. Importantly tumour differentiation is shown to be an independent prognostic factor. Patients recorded as well differentiated tumours had significantly better 3-year survival (61.3\%; $95 \%$ CI $53.2 \%$ to $68.4 \%$ ) compared to moderate $(38.4 \%$; $95 \%$ CI $33.2 \%$ to $43.6 \%$ ) and poor differentiation (34.1\%; $95 \%$ CI $30.1 \%$ to $38.1 \%$ - Abstract OC-104 figure 1. The effect remains significant after adjusting for age and gender with HR of 1.74 (95\% CI 1.11 to 2.71 ) for moderately differentiated and 2.1 (95\% CI 1.3 to 3.4 ) for poorly differentiated tumours compared to well differentiated tumour.

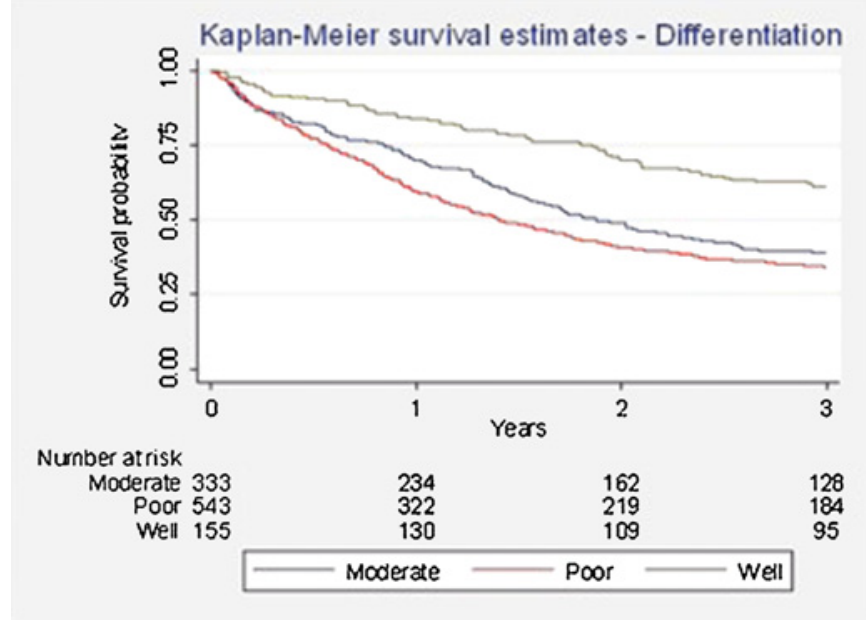

Abstract OC-104 Figure 1 Kaplan-Meier survival estimates for differentiation $(p<0.0001)$.

Conclusion The variables in the RCPath stomach cancer dataset are validated to be of prognostic significance in a population setting. Importantly, the result showed that there is a significant survival differences between the three grades of differentiation. This is in contrast to the current recommendation by the RCPath that well and moderately differentiated tumours be reported together as a single category. ${ }^{1}$ We recommend separating this category in the next version of the RCPath stomach cancer dataset guidelines. 
Competing interests None declared.

REFERENCE

1. Novelli M. Dataset for the Histopathological Reporting of Gastric Carcinoma. Standards and Datasets for Reporting Cancers. 2nd edn. The Royal College of

\section{OC-105 IMPACT OF AJCC 7TH EDITION TNM STAGING ON A HISTORICAL OESOPHAGO-GASTRIC CANCER RESECTION DATASET}

doi:10.1136/gutjnl-2012-302514a.105

${ }^{1} \mathrm{~A}$ M Reece-Smith, ' M P John,* ${ }^{2} \mathrm{Z}$ Chaudry, 'S L Parsons. 'Department of Oesophago-gastric surgery, Nottingham University NHS trust, Nottingham, UK; ${ }^{2}$ Department of Histopathology, Nottingham University NHS trust, Nottingham, UK

Introduction The revised AJCC $^{1}$ TNM staging systems for oesophageal and gastric cancer were published in 2010 and have been adopted in many units since that time. However, such changes in staging system can cause problems in comparing recent datasets to historical data as stage matching will no longer be consistent. We aimed to review historical data to determine the impact of updated scoring systems on our dataset, firstly to determine the proportion of patients changing stage groups and secondly to determine if survival was different between new and old systems.

Methods A database of gastro-oesophageal resections has historically accumulated patients with operative stage recorded using 6th edition TNM classification. With the help of a specialist pathologist these cases were reassessed to determine the revised TNM according to the 7 th edition. The survival of matched stage disease was compared using Kaplan-Meier analysis with log-rank test for statistical significance.

Results In a cohort of 358 patients 50 patients (14.0\%) changed stage. Twenty-four to a lower stage and 26 to higher stages as detailed in the Abstract OC-105 table 1 below. Stage 2 and 3 contained sufficient patients for survival analysis. Median survival was not reached for stage 2 in TNM6 and was 37.9 months in TNM7 ( $p=0.651$ ). In stage 3 the survival was 16.7 months in TNM6 and 17.3 months using TNM7 ( $p=0.786)$. Survival was not significantly different between editions.

Conclusion Fourteen percent of patients change stage using updated criteria. However, the impact of these changes on the median survival of patients in specified stage groups is small. Re-evaluating historical patient data will not greatly advise clinicians or patients regarding their prognosis but this data does help in the comparison of historical publications to current data.

Competing interests None declared.

\section{REFERENCE}

1. Ann Surg Oncol 2010;17:1721-4. Pathologists. 2007.

\section{OC-106 SERONEGATIVE VILLOUS ATROPHY - COELIAC DISEASE JUST HALF THE TIME?}

doi:10.1136/gutjnl-2012-302514a.106

${ }^{1} \mathrm{I}$ Aziz, ${ }^{1}{ }^{1} \mathrm{~K}$ E Evans, ${ }^{2} \mathrm{~S} S \mathrm{C}$ Cross, ${ }^{1} \mathrm{D}$ S Sanders. ${ }^{1}$ Department of Gastroenterology, Royal Hallamshire Hospital, Sheffield, UK; ${ }^{2}$ Department of Histopathology, Royal Hallamshire Hospital, Sheffield, UK

Introduction Villous atrophy (VA) in the presence of a positive coeliac serology (endomysial [EMA] and tissue transglutaminase [tTG] antibodies) is highly predictive of coeliac disease (CD). However, diagnostic challenges may arise in those where VA is associated with a negative coeliac serology.

Aims To study the aetiology of seronegative VA.

Methods One hundred patients presenting with seronegative VA (both EMA negative and tTG normal range) were prospectively and systematically investigated for $\mathrm{CD}$ and other known associations of VA. The history, immunoglobulins and histopathology were initially reviewed, followed if necessary by a combination of HLA typing, gluten challenge, repeat coeliac serology \& duodenal biopsies, and exclusion of infection/inflammatory bowel disease.

Results 59 women, 41 men, age range 16-92 years, median age 49 years. CD was present in $44 \%$ of cases, followed by gastrointestinal infections (18\%), severe duodenitis (4\%), crohn's disease $(3 \%)$, tuberculosis $(2 \%)$, HIV $(2 \%)$, drugs $(2 \%)$ and haematological disorders $(2 \%)$. In $23 \%$ an association was not found, although the majority normalised their small bowel histology on repeat biopsies $(17 / 23,74 \%)$. Reviewing the case with a specialist gastrointestinal (GI) histopathologist played an important role in up to $9 \%$ of cases, in terms of identifying a cause or help tailoring further investigations. All coeliac patients tested for HLA status were as expected positive for DQ2 or DQ8 haplotypes (n 37), in comparison to 22/47 $(47 \%)$ non-coeliacs $(\mathrm{p}<0.0001)$. A non-coeliac diagnosis was significantly more common in asian/african patients or those with clinical symptoms of abdominal pain, weight loss or nausea \& vomiting $(p<0.05)$. However, there was no difference in age, gender, baseline biochemistry or haematological blood tests between the two groups. Conclusion Close collaboration with a specialist GI histopathologist is useful in cases of seronegative VA. A cause will be found in $77 \%$ of patients, with CD accounting for almost half of all cases. In the $23 \%$ with no apparent cause, reassuringly $74 \%$ normalised their histology. The minority with persisting VA of unknown cause remain under active follow-up. The prevalence of a positive HLA in cases deemed to be non-coeliacs was greater than that of $25 \%$ as accepted for the general population. Many of these cases were due to gastrointestinal infections or an unknown cause, and subsequently normalised their histology (18/22). A threshold model for CD has recently been proposed and these patients may still belong to this disease spectrum-further work looking for intestinal antibody deposits in this group may shed light on this possibility.

Competing interests None declared.

\section{OC-107 MORPHOMETRIC ANALYSIS OF FIBROSIS AND INFLAMMATORY ACTIVITY IN RECURRENT HCV INFECTION POST LIVER TRANSPLANTATION}

Abstract OC-105 Table 1

doi:10.1136/gutjnl-2012-302514a.107

\begin{tabular}{lrr}
\hline Stage change TNM6 to TNM7 & $\mathbf{N}=$ & $\%$ \\
\hline Stage 4 to stage 3 & 14 & 3.9 \\
Stage 2 to stage 1 & 10 & 2.8 \\
Stage 1 to stage 2 & 13 & 3.6 \\
Stage 2 to stage 3 & 13 & 3.6 \\
No change & 308 & 74.8 \\
Total & 358 & 100.0 \\
\hline
\end{tabular}

${ }^{1,2} \mathrm{~S} M$ Noory, ${ }^{*}{ }^{1,2} \mathrm{R}$ Hajhosseiny, ${ }^{1,2} \mathrm{G}$ Noory, ${ }^{2} \mathrm{D}$ Joshi, ${ }^{2} \mathrm{~A}$ Quaglia. ${ }^{1}$ School of Medicine, King's College London University, London, UK; ${ }^{2}$ Institute of Liver Studies, King's College Hospital, London, UK

Introduction Hepatitis $\mathrm{C}$ virus (HCV) recurrence post liver transplantation (LT) is universal. Ishak score (IS) is widely used in the histological assessment of recurrent HCV. Smooth muscle actin (SMA) expression is a marker of hepatic stellate cells (HSC), 